\title{
Non-linear controls influence functions in an aircraft dynamics simulator
}

\author{
Nelson M. Guerreiro*a, James E. Hubbard, Jr. ${ }^{a}$, Mark A. Motter ${ }^{\mathrm{b}}$ \\ ${ }^{a}$ Univ. of Maryland/National Inst. of Aerospace, 100 Exploration Way, Hampton, VA, USA, 23666; \\ bASA Langley Research Center, Mail Stop 488, Hampton, VA, USA, 23666;
}

\begin{abstract}
In the development and testing of novel structural and controls concepts, such as morphing aircraft wings, appropriate models are needed for proper system characterization. In most instances, available system models do not provide the required additional degrees of freedom for morphing structures but may be modified to some extent to achieve a compatible system. The objective of this study is to apply wind tunnel data collected for an Unmanned Air Vehicle (UAV), that implements trailing edge morphing, to create a non-linear dynamics simulator, using well defined rigid body equations of motion, where the aircraft stability derivatives change with control deflection. An analysis of this wind tunnel data, using data extraction algorithms, was performed to determine the reference aerodynamic force and moment coefficients for the aircraft. Further, non-linear influence functions were obtained for each of the aircraft's control surfaces, including the sixteen trailing edge flap segments. These non-linear controls influence functions are applied to the aircraft dynamics to produce deflection-dependent aircraft stability derivatives in a non-linear dynamics simulator. Time domain analysis of the aircraft motion, trajectory, and state histories can be performed using these nonlinear dynamics and may be visualized using a 3-dimensional aircraft model. Linear system models can be extracted to facilitate frequency domain analysis of the system and for control law development. The results of this study are useful in similar projects where trailing edge morphing is employed and will be instrumental in the University of Maryland's continuing study of active wing load control.
\end{abstract}

Keywords: Influence functions, simulation, dynamics, UAV, morphing, stability derivatives, aerodynamic coefficients.

\section{INTRODUCTION}

In the development and testing of novel structural and controls concepts, such as morphing aircraft wings, appropriate models are needed for proper system characterization. These models provide a means for understanding the characteristics and behaviors of such concepts in real world applications, but may be difficult to derive. In this study, a well understood aircraft dynamics model was modified to represent an aircraft with non-conventional control surfaces.

Development of an aircraft dynamics simulation requires the gathering of information about the aircraft in question. Extensive wind tunnel data was used to obtain the aerodynamics force, moment, and damping derivatives for an Unmanned Aerial Vehicle (UAV) with main wing trailing edge segmented flaps. This data was used to create a component build up method, in the form of non-linear controls influence functions, for determining an aircraft's stability derivatives at any point in the simulation, allowing for stability derivatives that change with time and control deflection magnitude. These stability derivative models are implemented in a non-linear aircraft dynamics simulation.

The motivation for this paper comes from the need to understand the dynamics of aircraft with non-conventional control surfaces, such as morphing aircraft. These aircraft provide a wide range of new capabilities, in the form of additional degrees of freedom, which can be used to perform functions in addition to providing conventional control. The resulting aircraft dynamics simulation provides a multiple functionality tool that can be used for aircraft performance characterization and the extraction of system models for controls design. This study is in support of the University of Maryland's Lift Distribution Control (LDC) research. 


\section{BACKGROUND AND METHODOLOGY}

\subsection{Aircraft equations of motion}

The aircraft equations of motion used to build this model are well-defined equations. Stevens and Lewis ${ }^{1}$, and Pamadi ${ }^{2}$ provide two sources for in-depth understanding of aircraft dynamics and are left as references to the conventional aircraft dynamics notation used in this paper. The rigid-body non-linear equations of motion, as presented in Stevens and Lewis ${ }^{1}$, and referenced to the aircraft body-fixed frame, are given by equations (1) - (12).

Force equations:

$$
\begin{aligned}
& \dot{u}=r v-q w+g \sin \theta+\left(X_{A}+X_{T}\right) / M \\
& \dot{v}=-r u+p w+g \sin \phi \cos \theta+\left(Y_{A}+Y_{T}\right) / M \\
& \dot{w}=q u-p v+g \cos \phi \cos \theta+\left(Z_{A}+Z_{T}\right) / M
\end{aligned}
$$

Kinematic equations:

$$
\begin{aligned}
& \dot{\phi}=p+\tan \theta(q \sin \phi+r \cos \phi) \\
& \dot{\theta}=q \cos \phi-r \sin \phi \\
& \dot{\psi}=(q \sin \phi+r \cos \phi) / \cos \theta
\end{aligned}
$$

Moment equations:

$$
\begin{aligned}
& \dot{p}=\left(\frac{1}{I_{x} I_{z}-I_{x z}^{2}}\right)\left\{I_{x z}\left[I_{x}-I_{y}+I_{z}\right] p q-\left[I_{z}\left(I_{z}-I_{y}\right)+I_{x z}^{2}\right] q r+I_{x z} l+I_{x z} n\right\} \\
& \dot{q}=\left(\frac{1}{I_{y}}\right)\left\{\left(I_{z}-I_{x}\right) p r-I_{x z}\left(p^{2}-r^{2}\right)+m\right\} \\
& \left.\dot{r}=\left(\frac{1}{I_{x} I_{z}-I_{x z}^{2}}\right)\left\{\left(I_{x}-I_{y}\right) I_{x}+I_{x z}^{2}\right] p q-I_{x z}\left[I_{x}-I_{y}+I_{z}\right] q r+I_{x z} l+I_{x} n\right\}
\end{aligned}
$$

Navigation Equations:

$$
\begin{aligned}
& \dot{N}=u(\cos \theta \cos \psi)+v(-\cos \phi \sin \psi+\sin \phi \sin \theta \cos \psi)+w(\sin \phi \sin \psi+\cos \phi \sin \theta \cos \psi) \\
& \dot{E}=u(\cos \theta \sin \psi)+v(\cos \phi \cos \psi+\sin \phi \sin \theta \sin \psi)+w(-\sin \phi \cos \psi+\cos \phi \sin \theta \sin \psi) \\
& \dot{h}=u(\sin \theta)-v(\sin \phi \cos \theta)-w(\cos \phi \cos \theta)
\end{aligned}
$$

Linearized models are extracted from these non-linear equations and their standard form must be used. The equations of motion are rearranged and rotated into other coordinate frames, such as the wind coordinate frame, to organize the aircraft state vector into the standard aerospace form:

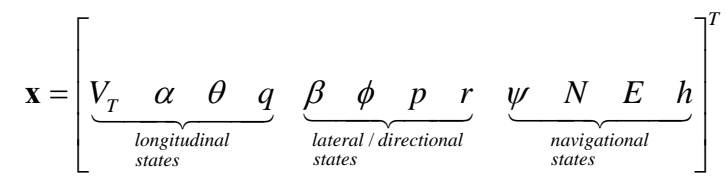

\subsection{Testbed aircraft}

This study is concerned with understanding the dynamics of a UAV with novel control effectors. The aircraft, a former US Army target drone classified as the FQM-117B, extensively modified, housed, and flown by NASA Langley Research Center (LaRC), implements segmented trailing edge flaps to achieve trailing edge morphing. Segmented trailing edge flaps consist of many independently controlled aileron segments that provide additional degrees of freedom in aircraft control. This one-of-a-kind aircraft is part of NASA LaRC's FLying Controls testbed (FLiC) program used for neural network and other advanced controller development and testing. Figure 1 shows these segmented flaps and their corresponding identification number. 


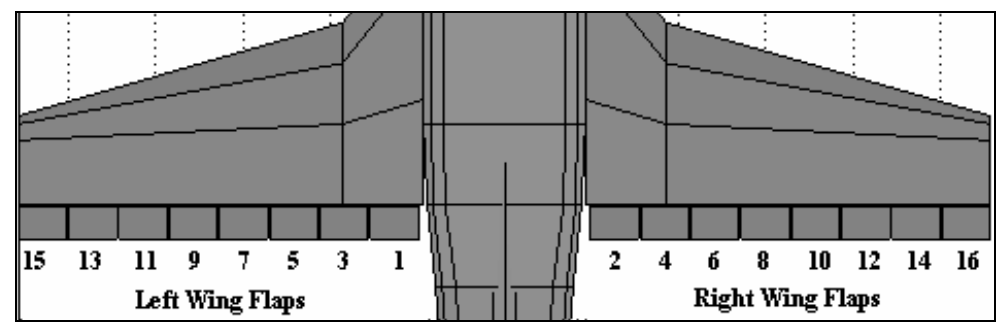

Figure 1: Segmented trailing edge flaps designations (top view).

\subsection{Wind tunnel data}

Two similar aircraft were wind tunnel tested in NASA Langley's 12-foot Low Speed Tunnel. These aircraft allowed for the testing of several configurations, such that a baseline reference of data could be obtained. Between the two aircraft, a total of 275 static wind tunnel test runs were performed.

One aircraft allowed for testing of a wing with full-span ailerons; each aileron surface encompassed one entire half wingspan. This same aircraft could also be equipped with conventional ailerons and flaps; each aileron surface accounted for one quarter wingspan, as did each flap surface.

A second aircraft configuration enabled testing of the segmented trailing edge flaps, with each aileron segment independently controlled. With respect to the remaining control surfaces, the two aircraft were virtually identical, with the exception of a slightly larger elevator control surface on one of the two.

The wind tunnel experiments were conducted at an average tunnel dynamic pressure of $2.95 \mathrm{lb} / \mathrm{ft}^{2}$. This correlates to a flow velocity of approximately 30 knots at sea level density. The typical airspeeds of the aircraft in question range from about 30 knots to 70 knots. A target airspeed of 45 knots was chosen as a reference for model development and data collection. This airspeed translates to a required lift coefficient of approximately 0.45 at an angle of attack of about 2 degrees. Thus, all influence coefficient data collected is referenced to a nominal 2 degree angle of attack data set.

\subsubsection{Data extraction algorithms}

Table 1: Matrix of wind tunnel run identifiers.

\begin{tabular}{|c|c|c|c|c|c|c|c|c|c|c|c|}
\hline \multirow[b]{2}{*}{ Input } & \multicolumn{11}{|c|}{ Deflections (Degrees) } \\
\hline & -25 & -20 & -15 & -10 & -5 & 0 & 5 & 10 & 15 & 20 & 25 \\
\hline Elevator & 79 & 78 & 77 & 76 & 75 & 54 & 80 & 81 & 82 & 83 & 84 \\
\hline Flap 1 & 0 & 134 & 133 & 132 & 131 & 120 & 135 & 136 & 137 & 138 & 0 \\
\hline Flap 3 & 0 & 142 & 141 & 140 & 139 & 120 & 143 & 144 & 145 & 146 & 0 \\
\hline Flap 5 & 0 & 150 & 149 & 148 & 147 & 120 & 151 & 152 & 153 & 154 & 0 \\
\hline Flap 7 & 0 & 158 & 157 & 156 & 155 & 120 & 159 & 160 & 161 & 162 & 0 \\
\hline Flap 9 & 0 & 166 & 165 & 164 & 163 & 120 & 167 & 168 & 169 & 170 & 0 \\
\hline Flap 11 & 0 & 174 & 173 & 172 & 171 & 120 & 175 & 176 & 177 & 178 & 0 \\
\hline Flap 13 & 0 & 182 & 181 & 180 & 179 & 120 & 183 & 184 & 185 & 186 & 0 \\
\hline Flap 15 & 0 & 190 & 189 & 188 & 187 & 120 & 191 & 192 & 193 & 194 & 0 \\
\hline Flap 2 & 0 & 202 & 201 & 200 & 199 & 120 & 195 & 196 & 197 & 198 & 0 \\
\hline Flap 4 & 0 & 210 & 209 & 208 & 207 & 120 & 203 & 204 & 205 & 206 & 0 \\
\hline Flap 6 & 0 & 218 & 217 & 216 & 215 & 120 & 211 & 212 & 213 & 214 & 0 \\
\hline Flap 8 & 0 & 226 & 225 & 224 & 223 & 120 & 219 & 220 & 221 & 222 & 0 \\
\hline Flap 10 & 0 & 234 & 233 & 232 & 231 & 120 & 227 & 228 & 229 & 230 & 0 \\
\hline Flap 12 & 0 & 242 & 241 & 240 & 239 & 120 & 235 & 236 & 237 & 238 & 0 \\
\hline Flap 14 & 0 & 250 & 249 & 248 & 247 & 120 & 243 & 244 & 245 & 246 & 0 \\
\hline Flap 16 & 0 & 259 & 258 & 257 & 256 & 120 & 251 & 252 & 254 & 255 & 0 \\
\hline Rudder & 89 & 88 & 87 & 86 & 85 & 54 & 90 & 91 & 92 & 93 & 94 \\
\hline Sideslip & 0 & 0 & 0 & 0 & 121 & 120 & 122 & 0 & 0 & 0 & 0 \\
\hline
\end{tabular}


The tremendous amount of data available in the form of wind tunnel runs led to the development of algorithms to automatically extract the data of interest. After determining the location of this data in the data set, a matrix of wind tunnel run indices was developed, which provided a map to the desired information. Functions were developed for the elevator, the rudder, the segmented flaps, and sideslip perturbations that would call the run matrix to obtain the wind tunnel run numbers. These functions extracted the data in question from the wind tunnel runs and formed the non-linear controls influence functions of interest. Table 1 shows the wind tunnel run matrix used to extract the desired information.

Some of the data extracted using this run matrix includes the reference coefficients for the aircraft as they vary with angle of attack and a unique algorithm was developed to extract this information. These reference coefficients are used to generate the traditional lift, drag, and moment profile curves for the aircraft. The influence functions can then be referenced with respect to these nominal values.

\section{MOTIVATION}

\subsection{Influence functions}

Flexibility and stiffness influence coefficients are typically associated with vibrations or structural dynamics. These coefficients are used to describe how a system behaves when subjected to loads. ${ }^{3}$ This is analogous to the aerodynamic and control derivatives used in aircraft dynamics. Typically, these coefficients are obtained at a reference or equilibrium point of interest of the system in question.

The large number of control flaps on this aircraft presents a challenge in determining how to model the influence of each surface. Previous research on an aircraft with a similar control flap arrangement indicated that linear superposition could be used to model the total influence on the aircraft dynamics. ${ }^{4}$ The influence functions developed here are implemented, using linear superposition, to create the overall system dynamics.

Consider a vector containing the aerodynamic coefficients for the aircraft at an un-accelerated attitude with un-deflected (centered) control surfaces, fixed airspeed, angle of attack and sideslip angle.

$$
\mathbf{a}_{0}=\left[\begin{array}{llllll}
C_{L} & C_{D} & C_{Y} & C_{l} & C_{m} & C_{n}
\end{array}\right]^{T}
$$

At this reference attitude, it can be assumed that any changes in the elements of $\mathbf{a}_{0}$ are due purely to control inputs. The vector of control inputs for this aircraft is as follows:

$$
\mathbf{u}=\left[\begin{array}{lllll}
\delta_{t} & \delta_{e} & \boldsymbol{\delta}_{\mathrm{la}} & \boldsymbol{\delta}_{\mathrm{ra}} & \delta_{r}
\end{array}\right]^{T}
$$

where,

$$
\begin{aligned}
\boldsymbol{\delta}_{\mathrm{la}} & =\left[\begin{array}{llllllll}
\delta_{a 1} & \delta_{a 3} & \delta_{a 5} & \delta_{a 7} & \delta_{a 9} & \delta_{a 11} & \delta_{a 13} & \delta_{a 15}
\end{array}\right] \\
\boldsymbol{\delta}_{\mathrm{ra}} & =\left[\begin{array}{llllllll}
\delta_{a 2} & \delta_{a 4} & \delta_{a 6} & \delta_{a 8} & \delta_{a 10} & \delta_{a 12} & \delta_{a 14} & \delta_{a 16}
\end{array}\right]
\end{aligned}
$$

and $t, e, l a, r a$, and $r$ subscripts represent the throttle, elevator, left aileron flaps, right ailerons flaps, and rudder, respectively. For notational purposes, denote the reference coefficients and the control vectors simply as:

$$
\mathbf{a}=\left[\begin{array}{llll}
a_{1} & a_{2} & \cdots & a_{k}
\end{array}\right]^{T} \quad \mathbf{u}=\left[\begin{array}{llll}
\delta_{1} & \delta_{2} & \cdots & \delta_{p}
\end{array}\right]^{T}
$$

where the indices $k=6$ and $p=19$ in this example. 
Perturb or deflect the control surfaces some arbitrary amount away from the un-deflected position and obtain the change in the coefficients. The change in the $i^{\text {th }}$ coefficient away from its reference values $a_{i_{0}}$ due to this arbitrary set of control deflections can be defined as follows:

$$
\Delta a_{i}=a_{i_{\text {new }}}-a_{i_{0}}
$$

Consider the contribution from each control surface independently and expand the expression for the change in the coefficient as a linear superposition of the changes due to the $p$ control flaps using partial derivatives, ignoring higher order terms:

$$
\Delta a_{i}=\frac{\partial a_{i}}{\partial \delta_{1}} \Delta \delta_{1}+\frac{\partial a_{i}}{\partial \delta_{2}} \Delta \delta_{2}+\cdots+\frac{\partial a_{i}}{\partial \delta_{p}} \Delta \delta_{p}=\sum_{j=1}^{p}\left(\frac{\partial a_{i}}{\partial \delta_{j}} \Delta \delta_{j}\right)
$$

Equation (14) provides the method for determining the resulting aerodynamic coefficients due to the control inputs:

$$
a_{i_{\text {new }}}=a_{i_{0}}+\sum_{j=1}^{p}\left(\frac{\partial a_{i}}{\partial \delta_{j}} \Delta \delta_{j}\right)
$$

The control inputs are referenced with respect to their un-deflected positions, $\delta_{j_{0}}=0$, therefore equation (15) becomes:

$$
a_{i_{\text {new }}}=a_{i_{0}}+\sum_{j=1}^{p}\left(\frac{\partial a_{i}}{\partial \delta_{j}} \delta_{j}\right)
$$

From equation (16), the influence coefficients are defined as the change in the aerodynamic coefficient $a_{i}$ resulting from a deflection of the control surface $\delta_{j}$ :

$$
c_{i, j}=\frac{\partial a_{i}}{\partial \delta_{j}}
$$

Equation (17) gives rise to some of the control derivatives traditionally used to describe aircraft dynamics. For example, the change in pitching moment coefficient due to a change in elevator control input is:

$$
C_{5,2}=\frac{\partial a_{5}}{\partial \delta_{2}}=\frac{\partial C_{m}}{\partial \delta_{e}}=C_{m_{\dot{x}}}
$$

This specific control derivative is known as the elevator control power.

Further, from equations (16) and (17), the linear controls influence functions can be defined as:

$$
b_{i, j}=f_{i}\left(\delta_{j}\right)=c_{i, j} \delta_{j}
$$


Traditionally, aerodynamic and control derivatives are collected for several flight conditions from either aircraft wind tunnel models, or from flight testing. Linearity is assumed and constant derivatives are used in describing the aircraft dynamics at or near the reference flight condition at which the derivatives were collected. This assumption can be considered valid only for small deflections of the control surfaces with small changes away from the reference flight condition.

Aircraft control surfaces may exhibit non-linear influences on the aircraft dynamics. This can be due to asymmetries in the positive and negative deflections of a specific surface or from other phenomenon, such as changes in the flow due to the control deflection. In an effort to help make the controls influences more accurate, the wind tunnel data here was used to formulate non-linear influence functions for each control surface. These models are implemented in the aircraft dynamics simulation as functions that are called at every instant in the calculations to determine the appropriate control derivatives for the specified control deflections.

The goal of this study was to develop functions that more accurately capture asymmetries in control deflections. Wind tunnel data was available that provided the capabilities to develop these functions for this specific aircraft. Trends were observed in the data and were used to develop function models that best represented each control input. The non-linear controls influence functions used to replace the linear functions are defined as:

$$
b_{i, j}=f_{i}\left(\delta_{j}, \delta_{j}^{2}, \cdots, \delta_{j}^{n}\right)
$$

where $b_{i, j}$ are polynomials of degree $n$ that represent best-fit trends to the wind tunnel data. Substituting equation (19) into equation (16) leads to the equation for the aerodynamic coefficients in terms of the non-linear controls influence functions.

$$
a_{i_{\text {new }}}=a_{i_{0}}+\sum_{j=1}^{p} b_{i, j}
$$

\subsubsection{Influence function models}

The first bits of information extracted from the available data were the aerodynamic force and moment coefficients for the aircraft. These coefficients describe the effects of angle of attack changes on the aircraft's aerodynamic forces and moments. $^{5}$ The aerodynamic force coefficients are the lift, drag, and side-force coefficients seen in Figure 2 . The moment coefficients are the rolling, pitching and yawing coefficients seen in Figure 3. The side-force, rolling moment, and yawing moment coefficients are small and have little influence on the aircraft dynamics with angle of attack changes, as can be seen in these figures. As a result, these coefficients are assigned zero reference values. The remaining coefficients are fitted using polynomials of the form:

$$
a_{i}(\alpha)=d_{n} \alpha^{n}+\cdots+d_{2} \alpha^{2}+d_{1} \alpha+d_{0}
$$

The values of the coefficients for these polynomial fits are listed in Table 2.

Table 2: Reference aerodynamic coefficient polynomial fits.

\begin{tabular}{lcccccccc}
\hline \hline Aero. Coefficient & $\boldsymbol{d}_{6}$ & $\boldsymbol{d}_{5}$ & $\boldsymbol{d}_{\mathbf{4}}$ & $\boldsymbol{d}_{3}$ & $\boldsymbol{d}_{\mathbf{2}}$ & $\boldsymbol{d}_{1}$ & $\boldsymbol{d}_{\boldsymbol{0}}$ & $\mathbf{R}^{2}$ \\
\hline Lift & - & - & - & $-1.12 \mathrm{E}-04$ & $2.03 \mathrm{E}-04$ & $7.90 \mathrm{E}-02$ & $-3.64 \mathrm{E}-01$ & 0.999 \\
Drag & - & - & - & - & $9.48 \mathrm{E}-04$ & $-2.46 \mathrm{E}-05$ & $3.67 \mathrm{E}-02$ & 0.988 \\
Pitching Mom. & $-3.82 \mathrm{E}-07$ & $0.00 \mathrm{E}+00$ & $9.44 \mathrm{E}-06$ & $-3.25 \mathrm{E}-05$ & $-8.05 \mathrm{E}-04$ & $9.17 \mathrm{E}-04$ & $2.39 \mathrm{E}-02$ & 0.998 \\
\hline
\end{tabular}




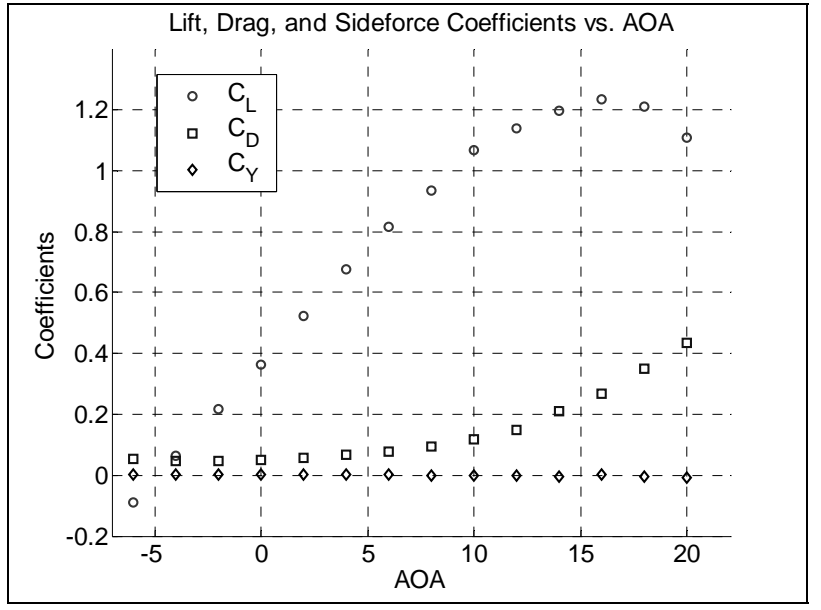

Figure 2: Force coefficients versus angle of attack.

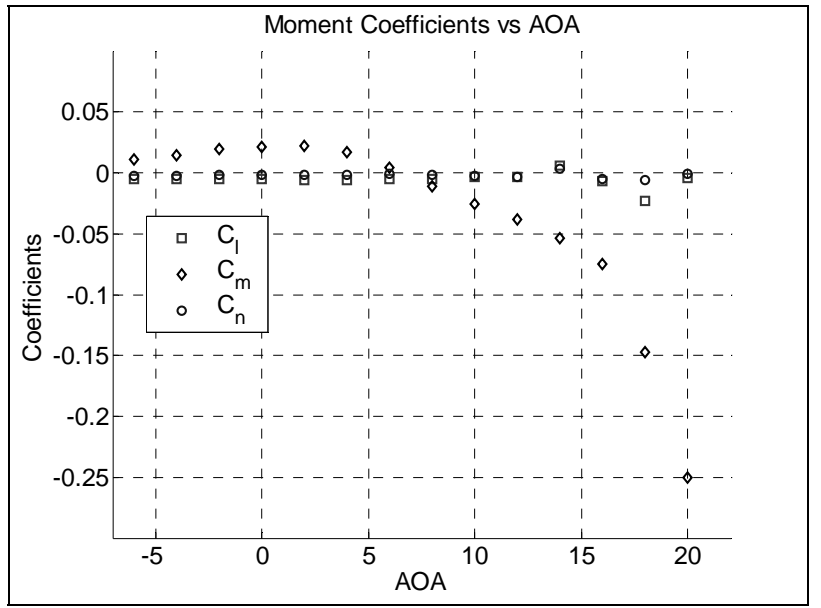

Figure 3: Moment coefficients versus angle of attack.

Changes away from the reference force and moment coefficients are due to the effects of the controls influence functions. Over 110 influence functions were extracted from the wind tunnel data and are used in describing the aircraft dynamics. Due to the large amount of data available and the limited space, only some of these representative influence functions are presented here.

The controls influence functions, as described in equation (19), are modeled with polynomial fits of the form:

$$
b_{i, j}\left(\delta_{j}\right)=d_{n} \delta_{j}^{n}+\cdots+d_{2} \delta_{j}^{2}+d_{1} \delta_{j}
$$

The influence function models that describe how the elevator inputs affect the reference aerodynamic coefficients are presented in Table 3.

Figure 4 and Figure 5 show the data used to fit these models. It is clear from the plots that three of the coefficients are not very sensitive to elevator deflections. These are the side-force coefficient, the rolling moment coefficient and the yawing moment coefficient. As a result, these are left as zero value influence functions.

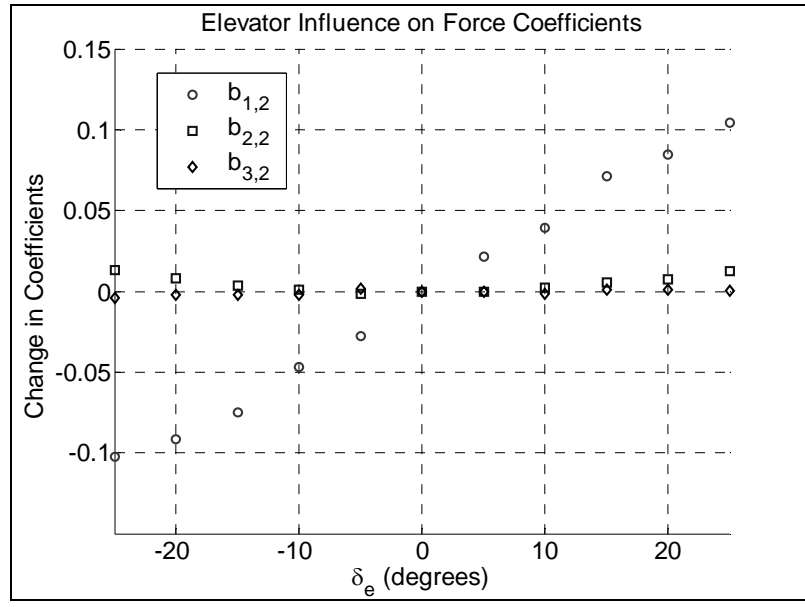

Figure 4: Elevator influence on the reference force coefficients.

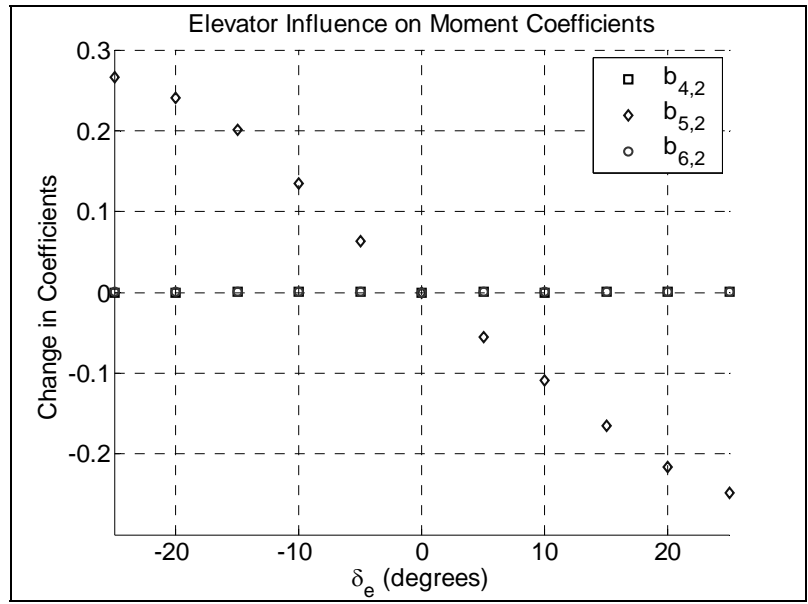

Figure 5: Elevator influence on the reference moment coefficients. 
Table 3: Elevator influence function models.

\begin{tabular}{ccccc}
\hline \hline Influence Function & $\boldsymbol{d}_{\mathbf{3}}$ & $\boldsymbol{d}_{\mathbf{2}}$ & $\boldsymbol{d}_{\mathbf{1}}$ & $\mathbf{R}^{\mathbf{2}}$ \\
\hline$b_{1,2}$ & $-1.16 \mathrm{E}-06$ & $-3.09 \mathrm{E}-06$ & $4.88 \mathrm{E}-03$ & 0.997 \\
$b_{2,2}$ & - & $1.99 \mathrm{E}-05$ & $1.04 \mathrm{E}-05$ & 0.968 \\
$b_{3,2}$ & - & - & $0.00 \mathrm{E}+00$ & - \\
$b_{4,2}$ & - & - & $0.00 \mathrm{E}+00$ & - \\
$b_{5,2}$ & $4.14 \mathrm{E}-06$ & $2.65 \mathrm{E}-05$ & $-1.29 \mathrm{E}-02$ & 0.998 \\
$b_{6,2}$ & - & - & $0.00 \mathrm{E}+00$ & - \\
\hline
\end{tabular}

The models for the rudder effectiveness use the same influence function form of equation (22). These models are listed in Table 4.

Table 4: Rudder influence function models.

\begin{tabular}{ccccc}
\hline \hline Influence Function & $\boldsymbol{d}_{\mathbf{3}}$ & $\boldsymbol{d}_{\mathbf{2}}$ & $\boldsymbol{d}_{\mathbf{1}}$ & $\mathbf{R}^{\mathbf{2}}$ \\
\hline$b_{1,19}$ & $3.14 \mathrm{E}-07$ & $-8.02 \mathrm{E}-06$ & $-3.21 \mathrm{E}-05$ & 0.379 \\
$b_{2,19}$ & - & $5.85 \mathrm{E}-06$ & $8.83 \mathrm{E}-06$ & 0.913 \\
$b_{3,19}$ & $-9.03 \mathrm{E}-08$ & $-1.03 \mathrm{E}-05$ & $1.54 \mathrm{E}-03$ & 1.000 \\
$b_{4,19}$ & - & - & $1.02 \mathrm{E}-04$ & 0.982 \\
$b_{5,19}$ & $2.57 \mathrm{E}-07$ & $5.34 \mathrm{E}-06$ & $-2.51 \mathrm{E}-05$ & 0.602 \\
$b_{6,19}$ & - & $5.15 \mathrm{E}-06$ & $-6.60 \mathrm{E}-04$ & 0.999 \\
\hline
\end{tabular}

Two of the rudder controls influence functions models do not seem to fit the data as close as desired, although they do not produce significant changes in the coefficients. These two are $b_{1,19}$ and $b_{5,19}$, which correspond to changes in the lift coefficient and changes in the pitching moment coefficient, respectively, due to rudder deflections. Changes in the lift coefficient may be due to disturbances of the flow over the horizontal tail, possible resulting in a small loss of the total aircraft lift. Also, changes in pitching moment coefficient would likely be due to increased drag resulting from a positive or negative rudder deflection. These factors and aero-elastic effects may be provoking these erratic influences.

Figure 6 and Figure 7 represent the data collected for the rudder influences. Evidence of asymmetric rudder influence can be seen in the function model for the yaw moment coefficient in Figure 7. Equal positive and negative deflections do not result in the same magnitude yawing moment coefficient. This asymmetry may be directly related to the asymmetric rudder geometry.

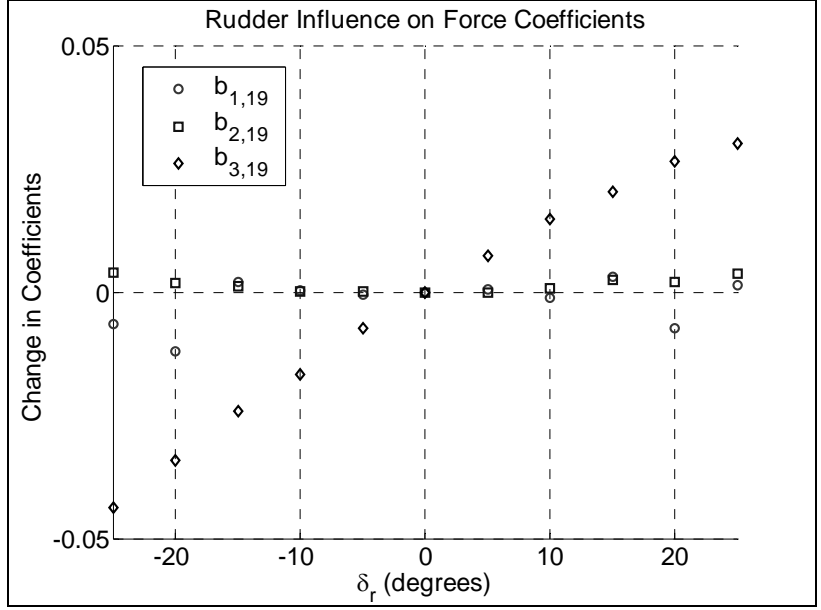

Figure 6: Rudder influence on the reference force coefficients.

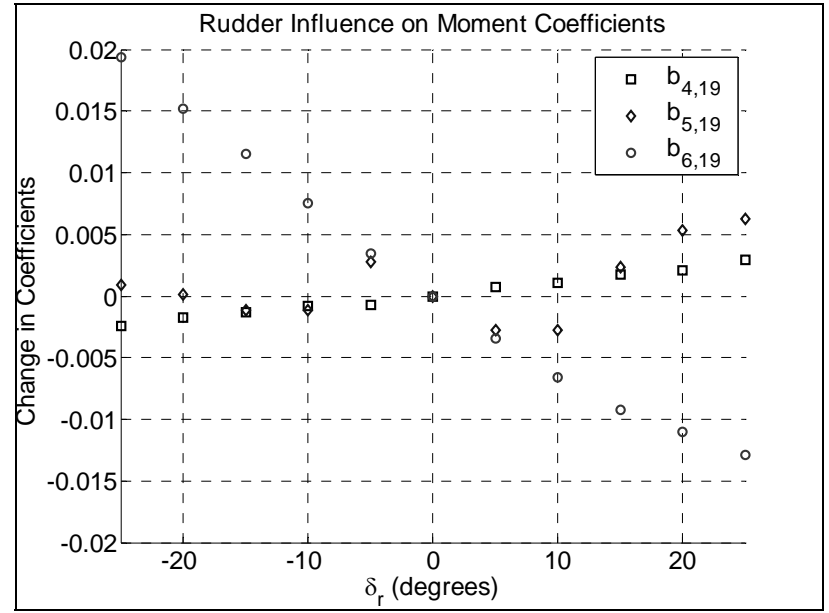

Figure 7: Rudder influence on the reference moment coefficients. 
The segmented aileron flaps influence functions presented next are for flap number 15, the left wing outboard flap, flap number 11, one of the left wing mid flaps, and flap number 1, the left wing flap at the wing root. These three flaps exhibit the same trends in the data and can be used to show inconsistencies in the flow properties related to the aerodynamic coefficient influences. The influence function models for these flap segments are presented in Table 5.

Table 5: Left wing sample flap (15, 11, and 1) influence function models.

\begin{tabular}{ccccc}
\hline \hline Influence Function & $\boldsymbol{d}_{3}$ & $\boldsymbol{d}_{\mathbf{2}}$ & $\boldsymbol{d}_{\mathbf{1}}$ & $\mathbf{R}^{\mathbf{2}}$ \\
\hline$b_{1,10}$ & $-6.84 \mathrm{E}-07$ & $2.06 \mathrm{E}-05$ & $6.54 \mathrm{E}-04$ & 0.685 \\
$b_{2,10}$ & - & $6.38 \mathrm{E}-06$ & $4.68 \mathrm{E}-05$ & 0.625 \\
$b_{3,10}$ & $-1.15 \mathrm{E}-07$ & $6.35 \mathrm{E}-06$ & $-3.68 \mathrm{E}-05$ & 0.797 \\
$b_{4,10}$ & $-5.37 \mathrm{E}-08$ & $1.92 \mathrm{E}-06$ & $2.65 \mathrm{E}-04$ & 0.988 \\
$b_{5,10}$ & $-2.04 \mathrm{E}-07$ & $1.38 \mathrm{E}-06$ & $-2.36 \mathrm{E}-04$ & 0.941 \\
$b_{6,10}$ & $---1.18 \mathrm{E}-06$ & $-1.43 \mathrm{E}-05$ & 0.929 \\
$b_{1,8}$ & $3.63 \mathrm{E}-07$ & $9.49 \mathrm{E}-06$ & $7.10 \mathrm{E}-04$ & 0.956 \\
$b_{2,8}$ & - & $3.67 \mathrm{E}-06$ & $2.30 \mathrm{E}-05$ & 0.689 \\
$b_{3,8}$ & $-1.82 \mathrm{E}-07$ & $-1.87 \mathrm{E}-06$ & $5.12 \mathrm{E}-05$ & 0.448 \\
$b_{4,8}$ & $-1.12 \mathrm{E}-08$ & $1.36 \mathrm{E}-06$ & $2.31 \mathrm{E}-04$ & 0.991 \\
$b_{5,8}$ & $1.60 \mathrm{E}-07$ & $3.74 \mathrm{E}-06$ & $-3.43 \mathrm{E}-04$ & 0.940 \\
$b_{6,8}$ & $------1.04 \mathrm{E}-06$ & $3.10 \mathrm{E}-06$ & 0.819 \\
$b_{1,3}$ & $5.01 \mathrm{E}-07$ & $1.12 \mathrm{E}-05$ & $5.69 \mathrm{E}-04$ & 0.882 \\
$b_{2,3}$ & - & $3.10 \mathrm{E}-06$ & $1.45 \mathrm{E}-05$ & 0.477 \\
$b_{3,3}$ & $-3.18 \mathrm{E}-07$ & $1.21 \mathrm{E}-06$ & $4.27 \mathrm{E}-05$ & 0.911 \\
$b_{4,3}$ & $1.11 \mathrm{E}-07$ & $1.35 \mathrm{E}-06$ & $7.06 \mathrm{E}-06$ & 0.937 \\
$b_{5,3}$ & $1.39 \mathrm{E}-08$ & $8.54 \mathrm{E}-07$ & $2.74 \mathrm{E}-04$ & 0.944 \\
$b_{6,3}$ & - & $4.63 \mathrm{E}-07$ & $6.95 \mathrm{E}-07$ & 0.518 \\
\hline & & & &
\end{tabular}

It is clear from the data presented in Figure 8 - Figure 13 that linear fits to this data may not accurately describe the influence of these flaps on the dynamics of the aircraft. The segmented flaps affect the lift coefficient in a general cubic curve-fit manner; the influences dropping off with increased deflections from nominal zero deflection. The contribution of these flaps to the drag coefficient shows a quadratic trend, where both positive and negative deflections provoke an increase in drag. The side-force coefficient, as expected, is not very sensitive to the deflection of the segmented aileron flaps.

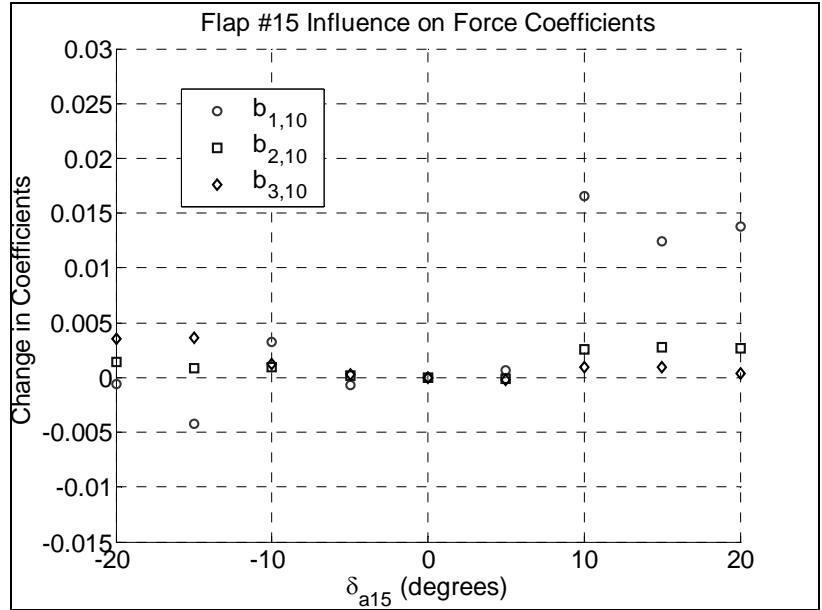

Figure 8: Flap number 15 effect on the reference force coefficients.

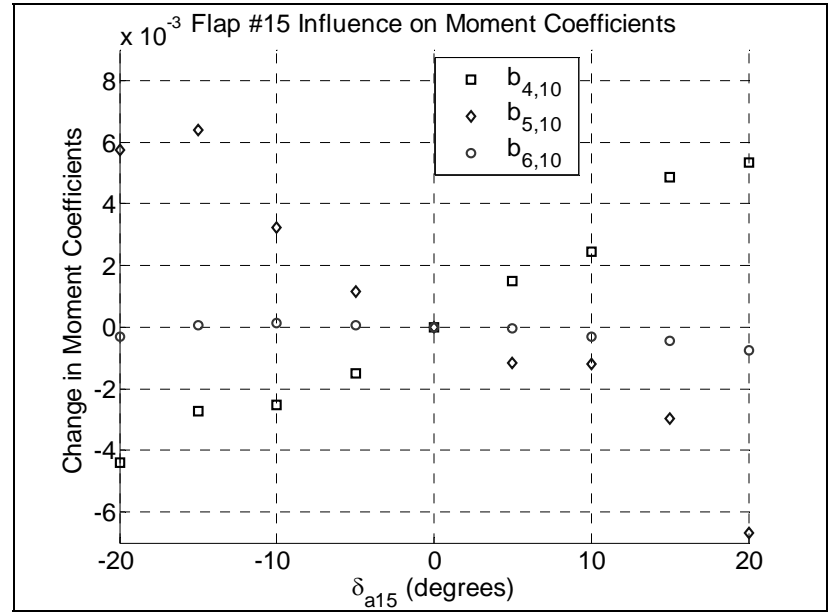

Figure 9: Flap number 15 effect on the reference moment coefficients. 


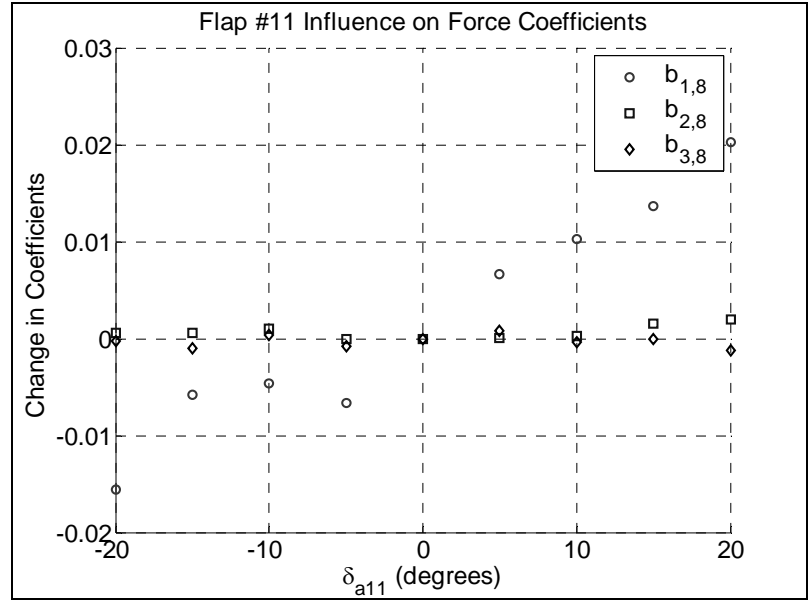

Figure 10: Flap number 11 effect on the reference force coefficients.

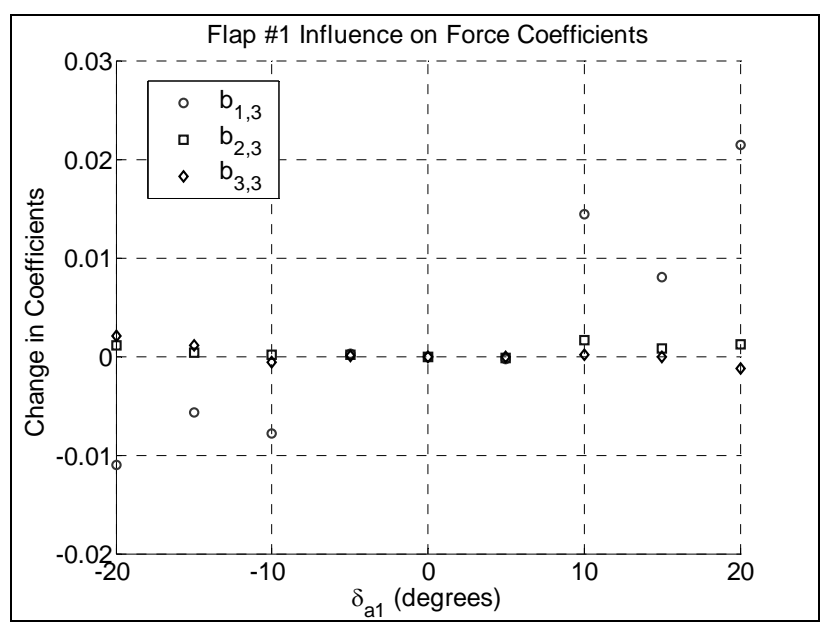

Figure 12: Flap number 1 effect on the reference force coefficients.

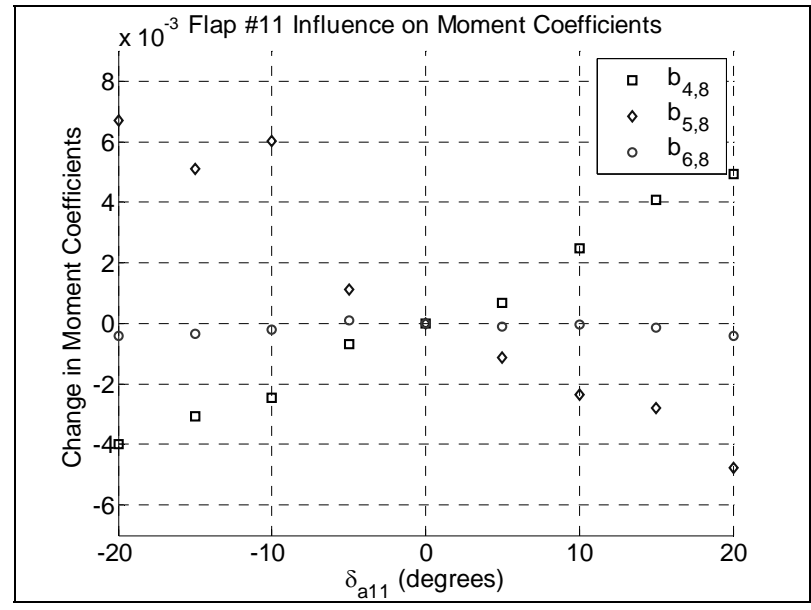

Figure 11: Flap number 11 effect on the reference moment coefficients.

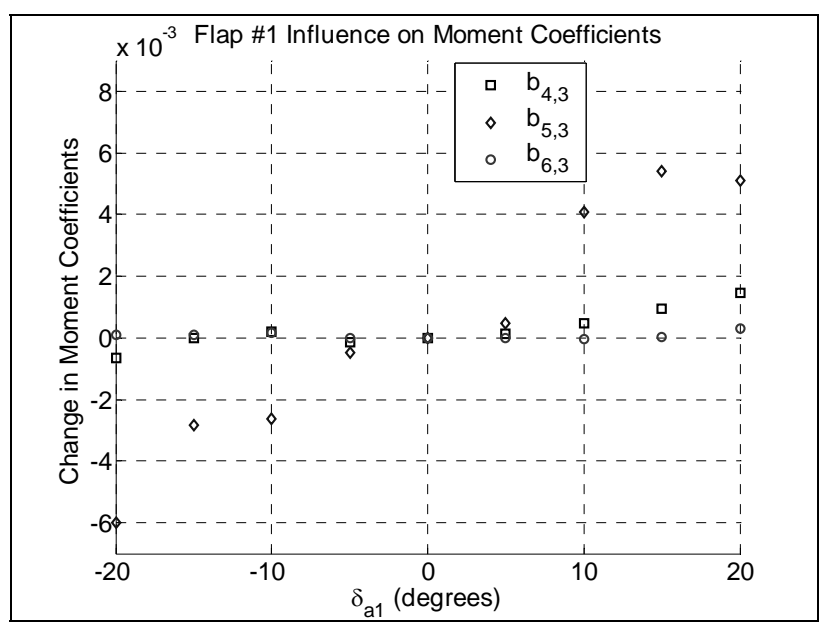

Figure 13: Flap number 1 effect on the reference moment coefficients.

The moment coefficient influence functions for these flaps show intuitive behavior and some possible flow inconsistencies. Positive deflections of these flaps should result in an increase in the rolling moment coefficient, while negative deflections should result in a decrease in this coefficient. The opposite is true for the right wing flaps. This trend is seen in the data presented in Figure 9, Figure 11, and Figure 13. It is interesting to note that this influence on the rolling moment shows another expected trend where the outboard flap, flap number 15, has a greater impact on the rolling moment than the inboard flap, flap number 1. Previous research, involving an aircraft with similar control surfaces, indicated this same trend with the inboard control flaps having relatively low impact on the roll moment. ${ }^{6}$

The yawing moment coefficient is not particularly sensitive to these flaps deflections but does show an expected trend. Deflections of these flaps, positive or negative, serve to increase the drag produced by that wing, causing an increase or decrease in the yawing moment. From this data, deflection of the left wing flaps will generally cause a decrease in the yawing moment coefficient, which is expected.

The pitching moment coefficient influence functions show some unexpected behavior that is consistent between the left and right wing segmented flaps. Figure 9 shows an increase in the pitching moment coefficient due to a negative 
deflection of flap segment number 15, and vice versa for a positive flap deflection. This is what is generally expected since a negative deflection of the flaps is a trailing edge up deflection and that would typically cause an increase in the pitching moment, or a nose-up pitching of the aircraft. As we look at flap number 11, moving along the left wing from the wingtip to the wing root, we see the same behavior or trend. Comparing this behavior to Figure 13, the influence of flap segment number 1 , we see a reversal in the pitching moment influence. This reversal in behavior is evident in the two inboard flap segments for each wing. Since these are the closest flaps to the fuselage, flow interactions may be occurring that result in this behavior.

The flap segment influence functions not presented here showed similar behavior to flaps 15 and 11 . All influence functions for the segmented trailing edge flaps have been implemented in the aircraft dynamics simulator.

Influence functions were also created for the sideslip effects on the force and moment coefficients. These influence function models were simple models because data was only available for -4 degrees, zero degrees, and 4 degrees sideslip deflections.

At this stage in the simulation development, influences on the aerodynamic coefficients due to throttle inputs will be assumed zero. This is not to say that these would not have an impact on the reference coefficients. In fact, this aircraft is a puller propeller aircraft and changes in throttle would lead to possible increases in lift coefficient due to additional flow velocity over the aircraft. Also, engine torque would likely influence the rolling moment coefficient.

\subsubsection{Additional models for simulation}

The influence functions developed here are static functions that describe the aircraft static stability. Additional modeling was required to ensure an accurate representation of the aircraft dynamics. In particular, damping was required in areas that did not yet have damping. Additionally, an engine model was needed for the aircraft.

Table 6 is a list of the stability derivatives or influence coefficients typically used to model aircraft dynamics. Some of these derivatives are already embedded in the model aircraft in the form of the influence functions. The lift-curve slope, for example is taken into account in the models extracted from the wind tunnel data for the reference aerodynamic coefficients.

Table 6: Longitudinal and lateral/directional aircraft stability derivatives.

\begin{tabular}{|c|c|c|c|c|c|}
\hline \multicolumn{3}{|c|}{ Longitudinal Stability Derivatives } & \multicolumn{3}{|c|}{ Lateral/Directional Stability Derivatives } \\
\hline Derivative & Description & Modeled As & Derivative & Description & Modeled As \\
\hline$C_{L_{\alpha}}$ & lift-curve slope & ref. coefficient models & $C_{l_{\beta}}$ & dihedral derivative & influence function \\
\hline$C_{m_{\alpha}}$ & pitch stiffness & ref. coefficient models & $C_{n_{\beta}}$ & yaw stiffness & influence function \\
\hline$C_{m_{q}}$ & pitch damping & estimation model & $C_{l_{p}}$ & roll damping & estimation model \\
\hline$C_{m_{V}}$ & tuck derivative & $\sim 0$ (subsonic) & $C_{n_{r}}$ & yaw damping & estimation model \\
\hline$C_{m_{\dot{\alpha}}}$ & alpha-dot derivative & $\sim 0$ (alpha-dot small) & $C_{n_{p}}$ & yaw moment due to roll rate & $\sim 0$ (assumed small) \\
\hline$C_{D_{v}}$ & speed damping & $\sim 0$ (low speed) & $C_{l_{r}}$ & roll moment due to yaw rate & $\sim 0$ (small yaw rate) \\
\hline$C_{D_{\alpha}}$ & drag-curve slope & ref. coefficient models & $C_{Y_{\beta}}$ & sideforce due to sideslip & influence function \\
\hline$C_{L_{V}}$ & lift vs. speed slope & $\sim 0$ (low speed) & $C_{Y_{r}}$ & sideforce due to yaw rate & $\sim 0$ (small yaw rate) \\
\hline$C_{L_{\dot{\alpha}}}$ & acceleration derivative for lift & $\sim 0$ (alpha-dot small) & $C_{Y_{p}}$ & sideforce due to roll rate & $\sim 0$ (assumed small) \\
\hline \multirow[t]{3}{*}{$C_{L_{q}}$} & pitch-rate dependent lift & $\sim 0$ (q small) & $C_{n_{\dot{\beta}}}$ & yaw mom. due to sideslip rate & $\begin{array}{l}\sim 0 \text { (small sideslip } \\
\text { rate) }\end{array}$ \\
\hline & & & $C_{Y_{\dot{\beta}}}$ & sideforce due to sideslip rate & $\begin{array}{l}\sim 0 \text { (small sideslip } \\
\text { rate) }\end{array}$ \\
\hline & & & $C_{l_{\dot{\beta}}}$ & $\begin{array}{l}\text { roll moment due to sideslip } \\
\text { rate }\end{array}$ & $\begin{array}{c}\sim 0 \text { (small sideslip } \\
\text { rate) }\end{array}$ \\
\hline
\end{tabular}

Many of the derivatives in Table 6 apply to high speed or high maneuverability aircraft and can be assumed to be zero. The aircraft in question is a low speed aircraft and, for the purposes of developing this simulation, it was assumed that 
the maneuvers performed are low speed or fairly benign. This assumption eliminates many of the derivatives from the list.

Given the assumptions of this model, three additional stability derivatives were modeled. These are the roll damping, pitch damping, and yaw damping derivatives. The pitch damping and yaw damping derivatives are statistical models that depend on the geometric properties of the aircraft. ${ }^{1,7}$ The roll damping derivative is an approximated guess that falls within historical levels and produces realistic dynamic behavior when compared to observed aircraft dynamics. These three damping derivative values are listed in Table 7.

Table 7: Damping derivatives.

\begin{tabular}{ccc}
\hline \hline Derivative & Description & Est. Value \\
\hline$C_{l_{p}}$ & roll damping & -0.25 \\
$C_{m_{q}}$ & pitch damping & -5.31 \\
$C_{n_{r}}$ & yaw damping & -0.50 \\
\hline
\end{tabular}

One additional model was developed for use in simulating the aircraft dynamics. Engine performance data was not available for this aircraft and a simple model was developed for the internal combustion, propeller driven engine. An estimate was made on the maximum static thrust of the engine and an exponential decay model was used to simulate a decrease in the available thrust with increasing airspeed.

In general, an aircraft dynamics simulation would also require an altitude density model. However, for purposes of the research at hand, all flights can be assumed to take place at or near sea level conditions, thus eliminating the need for this model.

\section{RESULTS}

\subsection{Simulation Capabilities}

The aircraft dynamics simulation developed using these non-linear controls influence functions results in a multiple capability tool. Of particular interest and usefulness is the ability to understand the dynamics of an aircraft that implements innovative controls surfaces for multifunctional purposes. Computer simulations allow for scenarios to be run and controllers to be tested prior to actual flight testing.

The simulation developed here will serve as a basis for continuing research. This model can easily be modified to account for changes in aircraft design by inputting new data obtained from either wind tunnel experiments or CFD analysis. Also, this model can easily be modified to incorporate aero-elastic effects.

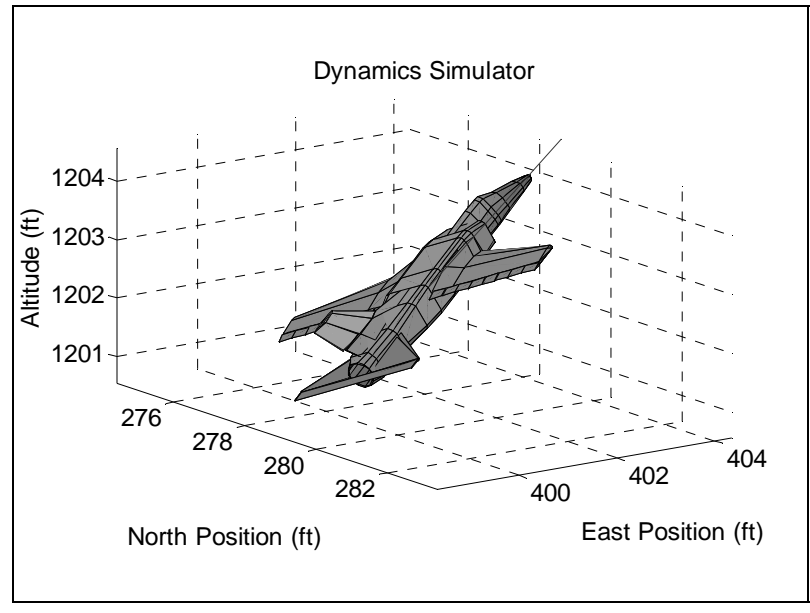

Figure 14: Dynamics visualization window.

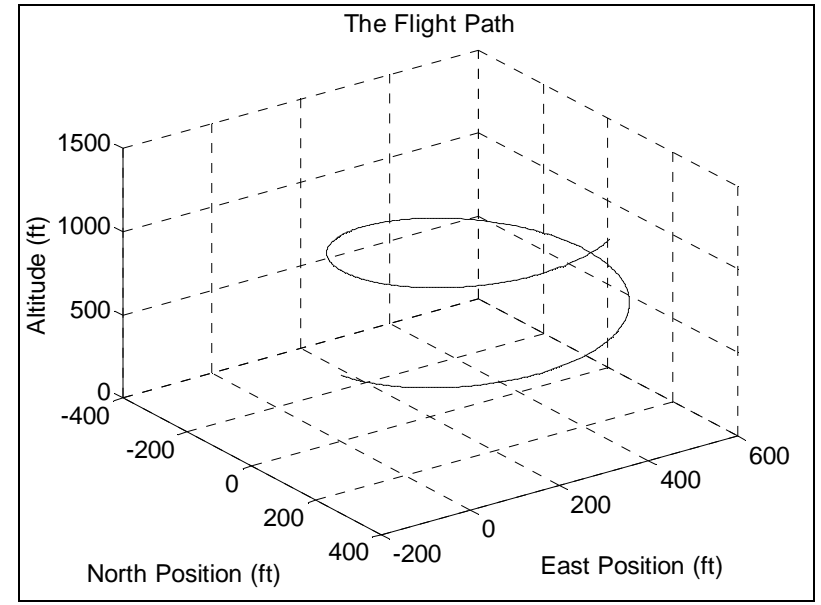

Figure 15: Aircraft trajectory visualization window. 
Another great capability of this aircraft dynamics model is the ability to extract linear plant models. A reference flight condition of interest can be chosen and a canonical form system model extracted for use in controller development. These linear models allow for the determination of system stability and frequency domain analysis. Control law developed using these linear models can easily be tested on the full non-linear aircraft model.

Free or controlled responses can be visualized via a 3-dimensional aircraft model translating and rotating in 3dimensional space as seen in Figure 14. The trajectory traveled by the aircraft during a specified maneuver can be seen in a similar 3-dimensional manner, as in Figure 15. Finally, the aircraft state time histories resulting from a maneuver can be analyzed from plots as can be seen in Figure 16.

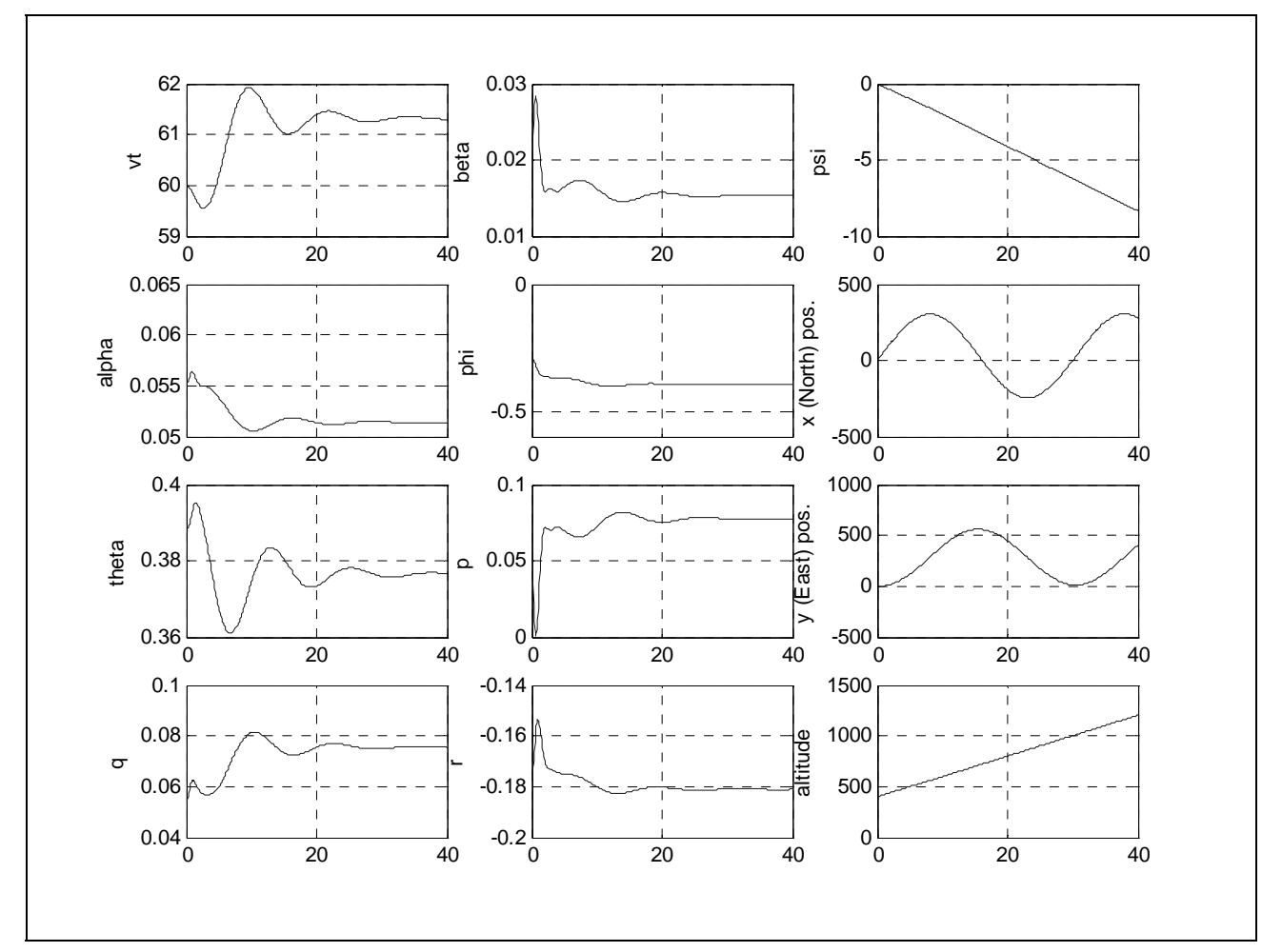

Figure 16: Aircraft states time histories (seconds).

In understanding the dynamics of an aircraft it is important to quantify its performance capabilities. The simulation developed here allows for the generation of a performance envelope for the aircraft, given the assumptions made in characterizing the dynamics. Information such as stall speed, control surface trim conditions, climb rate, and more can be extracted from data obtained from the simulation.

\section{CONCLUSIONS}

Non-linear controls influence functions have been implemented in a simulation to characterize the dynamics of an aircraft with novel controls effectors. The segmented trailing edge flaps on the aircraft in question provide additional degrees of freedom in control that could not be captured by traditional aircraft modeling techniques. The influence function method implemented in this study allows for control input asymmetries to be accurately represented in the aircraft dynamics.

As a result of this study, a dynamics model has been developed that allows for extraction of linear plant models and more. The model can be adapted with new influence functions if new wind tunnel data becomes available for the same aircraft or for an aircraft with a similar controls arrangement. The free or controlled response of the aircraft non-linear 
dynamics can be visualized in a 3-dimensional environment, in addition to presentation of the aircraft trajectory and the aircraft states time histories. Additional possibilities of this dynamics simulation can be endless and are left to the imagination of the reader.

\section{ACKNOWLEDGEMENTS}

The authors would like to thank NASA Langley Research Center, the National Institute of Aerospace, and the University of Maryland for their support in this research. Thanks also to the members of Morpheus Lab for their continued inspirational motivation.

\section{REFERENCES}

1. Stevens, B.L., Lewis, F.L., Aircraft Control and Simulation, $2^{\text {nd }}$ Edition, John Wiley \& Sons, Inc., Hoboken, NJ, 2003.

2. Pamadi, B.N., Performance, Stability, Dynamics, and Control of Airplanes $2^{\text {nd }}$ Edition, American Institute of Aeronautics and Astronautics, Inc., Reston, VA, 2004.

3. L. Meirovitch, Fundamentals of Vibrations, McGraw-Hill, New York, NY, 2001.

4. Abdulrahim, M., and Lind, R., "Investigating Segmented Trailing-Edge Surfaces for Full Authority Control of a UAV,” AIAA Atmospheric Flight Mechanics Conference, AIAA-2003-5312, Austin, TX, August 2003.

5. Anderson, J.D., Jr., Fundamentals of Aerodynamics, 3rd Edition, McGraw-Hill, New York, NY, 2001.

6. Abdulrahim, M., "Flight Dynamics and Control of an Aircraft With Segmented Control Surfaces," 42nd AIAA Aerospace Sciences Meeting and Exhibit, AIAA-2004-128, Reno, NV, January 2004.

7. Raymer, D.P., Aircraft Design: A Conceptual Approach, 3rd Edition, American Institute of Aeronautics and Astronautics, Inc., Reston, VA, 1999.

*Contact info: nelsong@umd.edu; phone 1757325 6832; fax 1757325 6701; www.umd.edu; www.nianet.org 